\title{
MicroRNA-33b downregulates the differentiation and development of porcine preadipocytes
}

\author{
Masaaki Taniguchi $\cdot$ Ikuyo Nakajima $\cdot$ \\ Koichi Chikuni · Misaki Kojima • Takashi Awata • \\ Satoshi Mikawa
}

Received: 11 February 2013/Accepted: 20 December 2013/Published online: 8 January 2014

(C) The Author(s) 2014. This article is published with open access at Springerlink.com

\begin{abstract}
Sterol regulatory element binding transcription factor (SREBF) is a key transcription regulator for lipid homeostasis. MicroRNA-33b (miR-33b) is embedded in intron 16 of porcine $S R E B F 1$ and is conserved among most mammals. Here, we investigated the effect of miR-33b on adipocyte differentiation and development in porcine subcutaneous pre-adipocytes (PSPA). PSPA were transiently transfected with miR-33b, and adipose differentiation was then induced. Delayed adipose differentiation and decreased lipid accumulation were observed in miR-33btransfected PSPA. Computational predictions suggested that miR-33b may target early B cell factor 1 (EBF1), an adipocyte activator of lipogenesis regulators such as CCAAT-enhancer binding protein alpha (C/EBP $\alpha)$ and peroxisome proliferator-activated receptor gamma $(\operatorname{PPAR} \gamma)$. Both gene and protein expression of EBF1 were downregulated in miR-33b-transfected PSPA, followed by considerable decreases in the expression of $C / E B P \alpha$ and $P P A R \gamma$ and their downstream lipogenic genes. However, miR-33b transfection did not markedly affect mRNA and protein expression of SREBF1. We also investigated differences in the expression of miR-33b and lipogenic genes
\end{abstract}

Electronic supplementary material The online version of this article (doi:10.1007/s11033-013-2954-z) contains supplementary material, which is available to authorized users.

M. Taniguchi $\cdot$ M. Kojima $\cdot$ T. Awata $\cdot$ S. Mikawa $(\bowtie)$ Animal Genome Research Unit, Agrogenomics Research Center, National Institute of Agrobiological Sciences, 2-1-2 Kannondai, Tsukuba, Ibaraki 305-8602, Japan

e-mail: mikawa@affrc.go.jp

I. Nakajima $\cdot$ K. Chikuni

Animal Products Research Division, National Institute of

Livestock and Grassland Science, 2 Ikenodai, Tsukuba,

Ibaraki 305-0901, Japan in subcutaneous fat tissues between 5-month-old crossbred gilts derived from Landrace (lean-type) and Meishan (fatty-type) boars. Landrace-derived crossbred gilts expressed more miR-33b and less lipogenic genes than did gilts derived from Meishan. Our results suggest that miR-33b affected the differentiation and development of PSPA by attenuating the lipogenic gene expression cascade through EBF1 to C/EBP $\alpha$ and PPAR $\gamma$. The differential expression of miR-33b observed in crossbred gilts may in part account for differences in lipogenic gene expression and the fat:lean ratio between pig breeds.

Keywords Adipocyte - Gene expression - Lipogenesis · MicroRNA $\cdot$ PPAR $\cdot$ SREBF

\section{Introduction}

Pork, together with chicken and beef, is an important protein source for humans. In the livestock industry, including pork production, carcass fat quantity and quality are major determinants of the productivity and palatability of meat. Previous work has shown that the molecular pathway of fat metabolism is regulated in a species-specific and fat-depot-specific manner [1, 2]. Therefore, it is essential to understand the molecular mechanisms that underlie adipogenesis and lipogenesis in porcine fat tissues.

Previous studies have investigated the transcriptional regulation of genes associated with adipogenesis [3, 4]. Among the transcription regulators, sterol regulatory element-binding transcription factor (SREBF) is known to regulate the transcriptional activation of genes involved in the uptake and synthesis of cholesterol, fatty acids, triglycerides, and phospholipids [5-7]. In addition to transcription factors, microRNAs (miRNA), which are $\sim 22$-nt 
non-coding RNAs generated from the sequential processing of long RNA transcripts [8], are considered to play key roles in the regulation of gene expression at the posttranscriptional level in the diverse regulatory pathways of many cellular processes. MirBase, a public database of miRNA, contains 331 miRNAs that have been identified in the porcine genome (Release 19 available since August 2012, http://www.mirbase.org) [9]. Recent studies [10,11] reported that miRNA (miR-33a) embedded in SREBF2 gene influences cholesterol metabolism in murine hepatocytes and human macrophages by repressing adenosinetriphosphate-binding cassette transporter A1 (ABCA1). In addition, Dávalos et al. [12] reported that miR-33a/b is associated with the repression of fatty acid oxidation and insulin signaling in hepatocytes. Further, identification and functional studies of miRNAs have been done in livestock species including pig and cattle [13, 14]. These studies suggest that fat cell development is achieved via a complicated mechanism that is potentially regulated by miRNAs in a species-specific manner.

Therefore, to clarify the porcine-specific pathways in adipocyte differentiation and development, we asked whether miR-33b influences the regulation of adipogenesis and lipogenesis in porcine preadipocytes. First, we determined the full-length nucleotide sequence of the porcine SREBF1 gene. Second, we transfected miR-33b into porcine subcutaneous pre-adipocytes (PSPA) to examine whether the transcriptional regulation of genes relevant to adipocyte differentiation and development was affected by miR-33b. We also investigated the mRNA and protein expression levels of an miR-33b target gene that was identified in an miRNA target prediction search and, as such, assumed to be involved in adipocyte differentiation and development. Last, we discuss the possible effect of miR-33b on differences in backfat (BF) thickness and blood triglyceride levels between representative lean and fat crossbred pigs.

\section{Materials and methods}

Animal samples

European crossbred (E) dams, including Landrace, Large White, and Duroc, were mated with Landrace (L) or Meishan (M) boars. Meishan pigs are substantially fatter, particularly in subcutaneous adipose tissue, than are typical European pig breeds $[15,16]$. Seven each of EL and EM gilts were fed under the same nutritional conditions until slaughter at 5 months old at the National Institute of Livestock and Grassland Science (NILGS). Body weights (mean $\pm \mathrm{SD}$ ) of the EL and EM at slaughter were $82.0 \pm 3.8$ and $82.8 \pm 6.2$, respectively.
There was no significant body weight difference between the breed types $(P=0.782$ by Student $t$ test). Tissue samples for total RNA extraction were collected from the liver, longissimus dorsi (LD) muscle, and subcutaneous adipose tissues at the mid-dorsal area of these animals. BF thickness measurements at the shoulder, back, and lumber positions were averaged and are presented as such. All animal care and use in this study was in accordance with the animal experimentation guideline of the NILGS and was approved by the NILGS Animal Care Committee.

\section{Cell culture}

PSPA were cultured in DMEM growth medium containing a low glucose concentration $(1.0 \mathrm{~g} / \mathrm{L})$ and $10 \%$ fetal bovine serum (FBS) (Invitrogen, Carlsbad, CA, USA). The subconfluent cells were passed every 3 days. To produce mature adipocytes, we plated the PSPA at $2.1 \times 10^{4}$ cells $/ \mathrm{cm}^{2}$ and grew them for 3 days to reach confluence. After the cells reached confluence (denoted as 0 day), adipose conversion was carried out in high-glucose $(4.5 \mathrm{~g} / \mathrm{L})$ DMEM containing $10 \%$ FBS, $5 \mu \mathrm{g} / \mathrm{mL}$ insulin (Sigma-Aldrich, Basel, Switzerland), $0.25 \mu \mathrm{M}$ dexamethasone (SigmaAldrich), $33 \mu \mathrm{M}$ biotin (Wako Pure Chemicals, Osaka, Japan), $17 \mu \mathrm{M}$ pantothenate (Sigma-Aldrich), and $5 \mathrm{mM}$ octanoate (Sigma-Aldrich). The medium was changed every other day. To investigate the effect of miR-33b on these cells, we subjected groups of cells to the following three experimental conditions: (1) growth medium alone; (2) differentiation with a negative control miRNA (miR-NC) transfection; and (3) differentiation with miR-33b transfection. All cells were cultured at $37{ }^{\circ} \mathrm{C}$ in a humidified incubator in $5 \% \mathrm{CO}_{2}$.

\section{Transfection}

To investigate the effect of miR-33b, we transfected a commercially available mature miRNA molecule, Pre$\mathrm{miR}^{\mathrm{TM}}$ miRNA Precursor (hsa-miR-33b) (Ambion, Austin, TX, USA), into confluent cells. The transfectant hsa-miR-33b is not a hairpin construct but a mature miRNA molecule, so that although it was designed for use with human cells, it can be applicable to pigs, cattle, or dogs, because the mature miRNA sequence of miR-33b is $100 \%$ identical among these species. For the miR-NC in experimental group 2, we used Pre-miR ${ }^{\mathrm{TM}}$ Negative Control \#1, which is a Pre-miR ${ }^{\mathrm{TM}}$ molecule designed to produce no identifiable effects on known miRNA function (Ambion). These transfectants were transfected by using the DharmaFECT 1 transfection reagent according to the manufacturer's instructions (ThermoFisher Scientific, Waltham, MA, USA). Four replicate samples of cells were harvested at 2, 
Table 1 Nucleotide sequences of primers and probes used for real-time PCR

\begin{tabular}{|c|c|c|}
\hline Gene & Nucleotide sequence of primers and probes $\left(5^{\prime}-3^{\prime}\right)$ & Accession number \\
\hline \multicolumn{3}{|l|}{ SREBF1 } \\
\hline Forward & CGGACGGCTCACAATGC & \multirow{3}{*}{ AB686492 } \\
\hline Reverse & GCAAGACGGCGGATTTATTC & \\
\hline Probe & TCAACGACAAGATCATCGAG & \\
\hline \multicolumn{3}{|l|}{$P P A R \gamma 1$} \\
\hline Forward & CTCGGACACCGGAGCTGG & \multirow{3}{*}{ AJ006756 } \\
\hline Reverse & CAACCATGGTCACCTCGCTAA & \\
\hline Probe & CGCCAGGCCACCACCGCAGATT & \\
\hline \multicolumn{3}{|l|}{$P P A R \gamma 2$} \\
\hline Forward & GGTGAAACTCTGGGAGATTCTCTTA & \multirow{3}{*}{ AF059245 } \\
\hline Reverse & CAACCATGGTCACCTCTTGTGA & \\
\hline Probe & CGATGCCTTCGACACGCTGTCTGCAA & \\
\hline \multicolumn{3}{|l|}{$C / E B P \alpha$} \\
\hline Forward & AGGAGGACGAGTCGAAGCA & \multirow{3}{*}{ XM_003127015 } \\
\hline Reverse & GGCGGAGGGTGTGAATGC & \\
\hline Probe & CTTTCCCTACCAGCCACCGCCGC & \\
\hline \multicolumn{3}{|l|}{$E B F 1$} \\
\hline Forward & ATGTTTGTCCATAATAACTCCAAGC & \multirow{3}{*}{ XM_003359834 } \\
\hline Reverse & CTTTGATACAGGGAGTAGCATGTT & \\
\hline Probe & ACCCCTCGGAAGGTACGCCCTCTTATC & \\
\hline \multicolumn{3}{|l|}{ FASN } \\
\hline Forward & GCTGGCCTACACGCAGAG & \multirow{3}{*}{ NM_001099930 } \\
\hline Reverse & GGCCCTGGAGCGGTATCA & \\
\hline Probe & CGCCTCCAGCACCTTGCCTTGC & \\
\hline \multicolumn{3}{|l|}{$a P 2$} \\
\hline Forward & CAGGAATTTGATGAAGTCACTGC & \multirow{3}{*}{ NM_001002817 } \\
\hline Reverse & GTGGTTGTCTTTCCATCCCAC & \\
\hline Probe & TGACAGGAAAGTCAAGAGCACCATAACCTT & \\
\hline \multicolumn{3}{|l|}{$A D I P O Q$} \\
\hline Forward & CACCACTGGCAAATTCCACTG & \multirow{3}{*}{ NM_214370 } \\
\hline Reverse & ССТTCACATCСТTCAAGTAGACC & \\
\hline Probe & CCTGGGCTGTACTACTTCTCCTTCCACG & \\
\hline \multicolumn{3}{|l|}{$C D 36$} \\
\hline Forward & CCTACTGGCTGAGTTATTGTGAC & \multirow{3}{*}{ NM_001044622 } \\
\hline Reverse & CACAGCATAGATTGACCTGCAA & \\
\hline Probe & TGGTACAGATGCAGCCTCATTTCCACCT & \\
\hline \multicolumn{3}{|l|}{$S C D 1$} \\
\hline Forward & ACGGATATCGCCCTTATGACAAG & \multirow{3}{*}{ NM_213781 } \\
\hline Reverse & CGCTGGCAGAATAGTCATAGGG & \\
\hline Probe & TGGAAGCCCTCACCCACAGCTCCC & \\
\hline
\end{tabular}

4, 8, 12, and 16 days after transfection and induction of adipocyte differentiation.

Cloning of the porcine SREBFl gene

A porcine bacterial artificial chromosome (BAC) library constructed from a boar of Large White/Landrace/Duroc composite was screened by means of a PCR-based method with primers derived from the porcine SREBF1 mRNA sequence (GenBank ID: NM_214157.1). BAC clones were sequenced to determine the full-length porcine SREBFI gene in accordance with the previously established method [17].

Gene expression assay

Total RNA including small RNA was extracted from porcine tissue samples and PSPA by using ISOGEN II 
(NIPPON GENE, Toyama, Japan). Total RNAs were reverse-transcribed to synthesize single-strand DNA by using ReverTra Ace (TOYOBO, Osaka, Japan). Real-time PCR was performed with the TaqMan system (Applied Biosystems, Foster City, CA, USA) to examine the relative gene expression of $S R E B F 1, C / E B P a, E B F 1$, fatty acid synthase $(F A S N)$, peroxisome proliferator-activated protein gamma 1 and $2(P P A R \gamma 1$ and PPAR 2$)$, stearoyl-CoA desaturase 1 ( $S C D 1)$, adipocyte-fatty acid binding protein $(a P 2)$, adiponectin $(A D I P O Q)$, and fatty acid translocase $(C D 36)$ by using primers and gene-specific probes (Table 1). The TaqMan MicroRNA Reverse Transcription Kit and TaqMan MicroRNA Assays (Applied Biosystems) designed for hsa-miR-33b were used for relative quantification of miR-33b. The TaqMan Endogenous Control Eukaryotic 18S rRNA gene (Applied Biosystems) was used for the relative quantification of all of the genes examined. The comparative threshold cycle method $(\Delta \Delta \mathrm{Ct})$ was employed to calculate the relative quantification of gene expression based on the formula, $2^{(-\Delta \Delta \mathrm{Ct})}$ where

$$
\begin{aligned}
\Delta \Delta \mathrm{Ct}= & \left(\mathrm{Ct}_{\text {targert gene }}-\mathrm{Ct}_{18 S r R N A}\right)_{\text {test }}-\left(\mathrm{Ct}_{\text {targert gene }}\right. \\
& \left.-\mathrm{Ct}_{18 S r R N A}\right)_{\text {calibrator }}
\end{aligned}
$$

$[18,19]$. The $\Delta \Delta \mathrm{Ct}$ values obtained from 0 day, non-treated control PSPA of experimental group 1 and from LD muscle were used as calibrators for the relative quantification of gene expression in the PSPA and 5-month-old gilts, respectively.

\section{Western blot analysis}

Nuclear and cytoplasmic proteins were prepared by using the CelLytic NuCLEAR Extraction Kit (Sigma-Aldrich), according to the manufacturer's instructions. The protein content was determined by using a bicinchoninic acid protein assay (Pierce, Rockford, IL, USA). Fifteen micrograms of nuclear or cytoplasmic protein were separated by electrophoresis through $12.5 \%$ SDS-polyacrylamide gels (ATTO, Tokyo, Japan). After they were electro-transferred onto nitrocellulose membranes by using the iBlot gel transfer system (Invitrogen), the proteins underwent Ponceau $\mathrm{S}$ staining (Sigma-Aldrich) to verify equal loading of the lanes. The membranes were then blocked overnight at $4{ }^{\circ} \mathrm{C}$ in phosphate buffered saline (PBS) containing $5 \%$ skim milk and $0.1 \%$ Tween 20 , followed by a 1 -h incubation at room temperature with primary polyclonal antibodies specific to EBF1 (Abcam, Cambridge, UK), the transcriptionally active form of SREBF1 in the nucleus (Abnova, Aachen, Germany), and $\beta$-actin (ACTB) (AnaSpec, San Jose, CA, USA). Horseradish peroxidase-conjugated anti-rabbit antibodies (GE Healthcare, Buckinghamshire, UK) served as secondary antibodies. Antigen-antibody complexes were visualized by use of the
ECL detection system (GE Healthcare), and the chemiluminescence signal was scanned with a LAS-3000 imaging system and quantified with Multi Gauge ver2.0, with which the imaging system was equipped (Fujifilm, Tokyo, Japan).

Lipid accumulation

At each time point of the culture period, PSPA were washed with PBS, fixed with $10 \%$ formaldehyde, and stained with filtered oil-red $\mathrm{O}$ solution $(0.3 \%$ oil-red $\mathrm{O}$ in $60 \%$ isopropanol). The triglycerides in the PSPA and blood from the crossbred gilts were extracted with chloroform-methanol $(2: 1, \mathrm{v} / \mathrm{v})$ and quantified enzymatically by using the Triglyceride E Test (Wako).

\section{Data analysis}

Measurements of gene expression, protein expression and triglyceride (TG) content were repeated in three independent experiments. Differences in gene expression, protein expression, and TG content among the cell culture treatments were analyzed with Tukey's multiple comparison tests $(P<0.05)$. Body weight, gene expression levels, $\mathrm{BF}$ thickness, and blood triglyceride concentration were compared between crossbred gilts by using the Student $t$ test $(P<0.05)$.

\section{Results and discussion}

Characterization of the full-length sequence of the porcine $S R E B F 1$ gene

First, we determined the 20,099-nt sequence of the fulllength porcine SREBF1 gene including the $5^{\prime}$-untranslated region (UTR), the 19 exons that encode the 3,456-nt open reading frame, the 18 introns, and the $3^{\prime}$-UTR (GenBank ID: AB686492). Unlike the human SREBF1 gene (GenBank ID: NG_029029.1), the porcine SREBF1 gene sequence that we determined, together with NCBI reference sequence (RefSeq) information, revealed that there is only one SREBF1 isoform, and no transcriptional variants have been identified in the pig genome (GenBank ID: NM_214157.1) or in cattle [20]. Porcine SREBF1 shares 86 and $83 \%$ homology to the human SREBF1c mRNA and amino acid sequences, respectively. In the full-length porcine SREBF1 gene sequence which we determined in this study, intron 16 included a sequence that is highly conserved among mammals and contains a pre-miRNA sequence (GenBank ID: AB686493) (Supplementary Fig. 1), while the corresponding region in recently published porcine genome sequence (NW_003540979) is not 


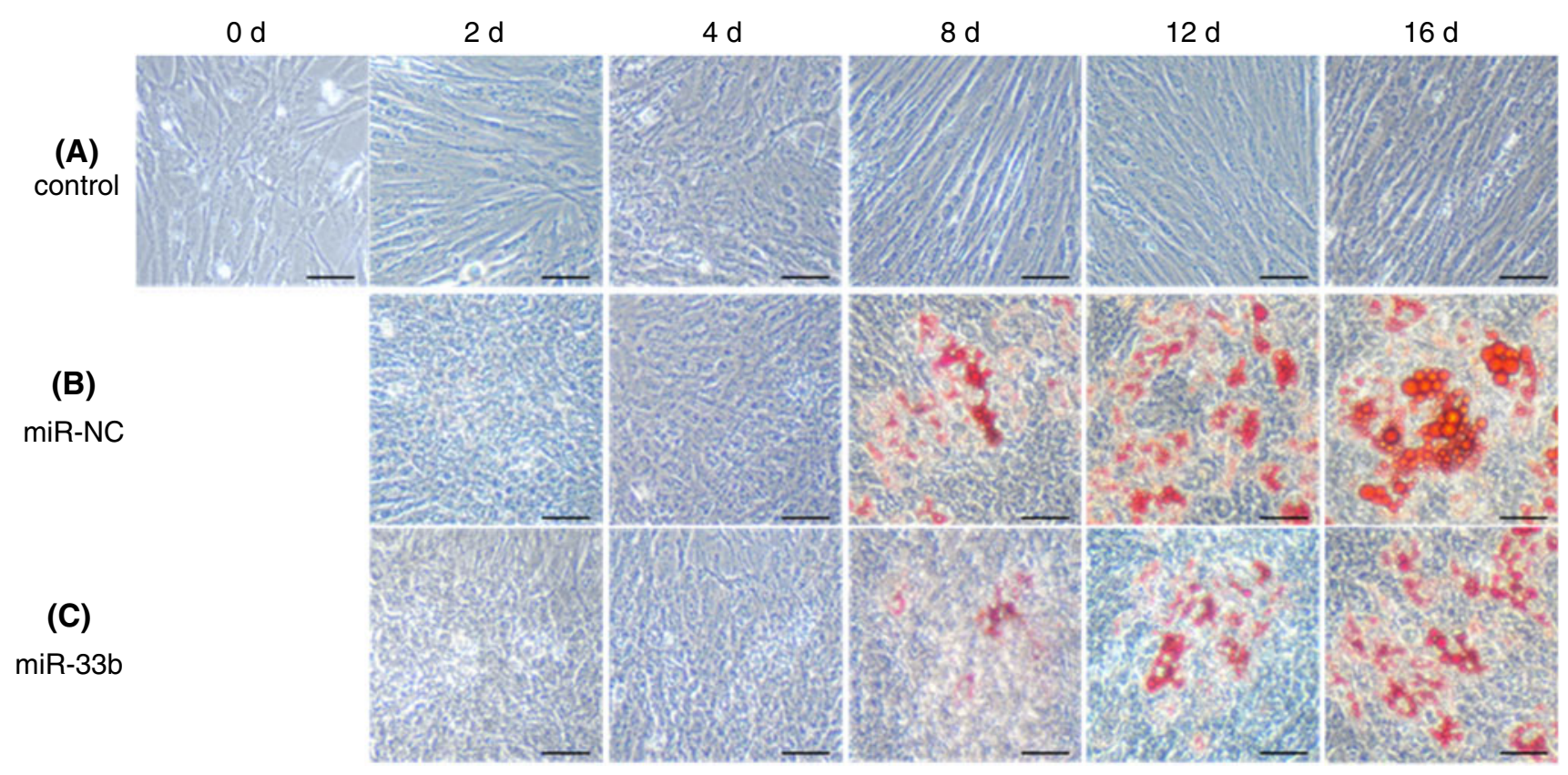

Fig. 1 Morphological characterization of PSPA. PSPA were treated with: a growth medium; b differentiation medium with negativecontrol miRNA (miR-NC) transfection; c Differentiation medium

identified. The pre-miRNA sequence expressed mature porcine miR-33b (ssc-miR-33b), which shared high homology with several other mammalian species except for mouse (Supplementary Fig. 1).

Effect of miR-33b on lipid accumulation in PSPA

To investigate the effect of miR-33b on lipid homeostasis, we transfected miR-33b into PSPA, which represent an ideal experimental model to study porcine adipocyte physiology [21]. Non-transfection/non-differentiation-induced control cells continued to proliferate and showed no signs of morphological change or lipid accumulation throughout the time course, whereas differentiation induction clearly induced lipogenesis after 4 days (Fig. 1). Transfection of miR-33b at differentiation induction decreased lipid accumulation in PSPA after 8 days compared with transfection of miR-NC (negative control microRNA), suggesting that miR-33b affected the differentiation and development of the PSPA (Fig. 1). Consistent with the observation of lipid accumulation by oil-red $\mathrm{O}$ staining, the TG content of the miR-33b-transfected cells was significantly $(P<0.05)$ decreased by $70.3 \%$ (4 days), $71.4 \%$ (8 days), $67.6 \%$ (12 days) and $77.4 \%$ (16 days) compared with that of the miR-NC-transfected cells (Fig. 2). These results clearly demonstrate that miR-33b attenuates the differentiation and development of PSPA. with miR-33b transfection. Lipid accumulation was visualized with oil-red O staining. Scale bar $50 \mu \mathrm{m}$

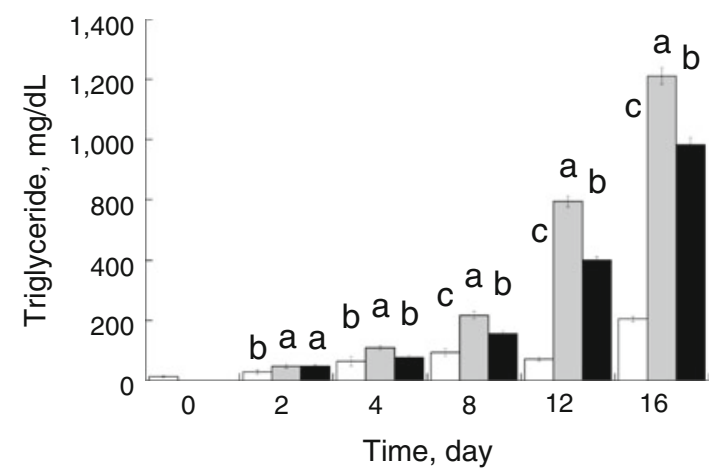

Fig. 2 Differences in triglyceride content in PSPA. PSPA were treated as follows: growth medium (white), differentiation medium with negative-control miRNA transfection (shaded), and differentiation medium with miR-33b transfection (black). Triglyceride content was measured at least three times. Comparisons of relative gene expression were performed by using Tukey's multiple comparison tests. Different letters show a significant difference $(P<0.05)$

\section{MiR-33b target gene prediction}

To investigate the biological function of miR-33b in PSPA, we explored putative miR-33b target genes by using algorithms including PITA [22], TargetScan v6.0 [23], and MicroRNA.org [24]. These computational predictions suggested that miR-33b may target porcine early B-cell factor 1 (EBF1) mRNA, because multiple possible miR-33b recognition sites were found in the $3^{\prime}$-UTR of porcine $E B F 1$ 

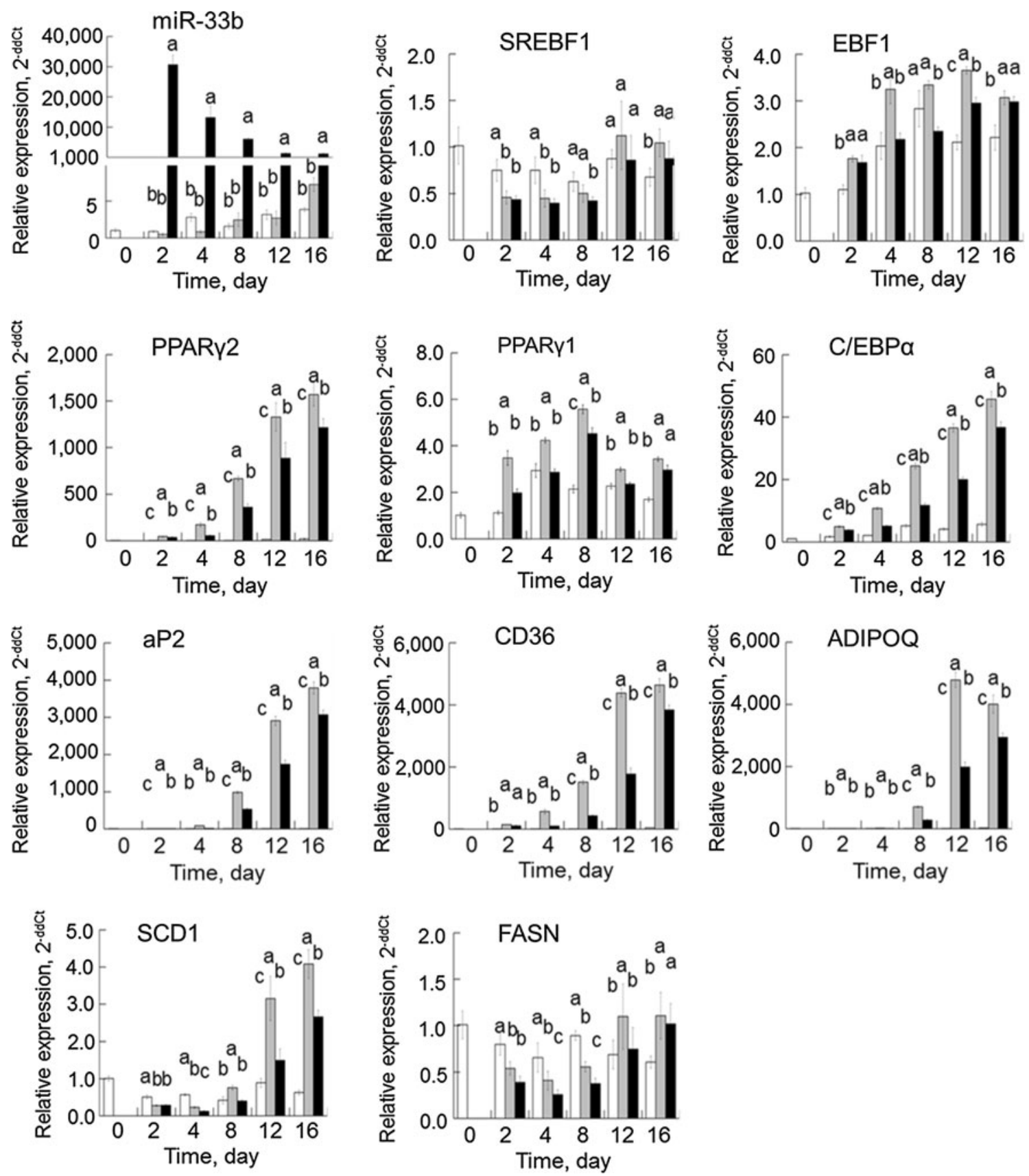

Fig. 3 Changes in adipogenic and lipogenic genes in PSPA. PSPA were treated as follows: growth medium (white), differentiation medium with negative-control miRNA transfection (shaded), and differentiation medium with miR-33b transfection (black). Relative

mRNA, one of which was $100 \%$ identical to that of numerous other mammalian species (Supplementary Fig. 2a, b). In contrast, none of the master regulators of adipocyte differentiation and development were predicted to be miR$33 \mathrm{~b}$ targets with these methods. These findings suggest that ssc-miR-33b has a conserved effect on the post-transcriptional regulation of EBF1 expression in porcine adipocytes. The BioSystems Database describing "Transcriptional Regulation of White Adipocyte Differentiation" in the gene expression was measured by using the $2^{-\Delta \Delta C t}$ method at least three times. Comparisons of relative gene expression were performed by using Tukey's multiple comparison tests. Different letters show a significant difference $(P<0.05)$

NCBI [24] suggested that EBF1 binds and activates the $P P A R \gamma$ promoter (http://www.ncbi.nlm.nih.gov/biosystem s?term=205243). In addition, Jimenez et al. [26] reported that EBF1 promotes adipogenesis by inducing the expression of the $C / E B P \alpha$ and $P P A R \gamma 1$ promoters, and repressing GATA-2, which is considered to negatively affect adipogenesis in mice [4]. Like SREBF1, C/EBP $\alpha$ and PPAR $\gamma$ are master regulators that are recognized as molecular markers of adipocyte differentiation and development [27-29]. 
(A)

\begin{tabular}{|c|c|c|c|c|c|c|c|c|c|c|c|c|c|c|c|c|c|}
\hline \multirow{2}{*}{ Cell treatment } & \multicolumn{16}{|c|}{ Time, day } & \multirow[b]{2}{*}{$\mathrm{H}^{1}$} \\
\hline & 0 & & 2 & & & 4 & & & 8 & & & 12 & & & 16 & & \\
\hline $\begin{array}{l}\text { Adipocyte } \\
\text { Differentiation }\end{array}$ & - & - & + & + & - & + & + & - & + & + & - & + & + & - & + & + & - \\
\hline $\begin{array}{l}\text { miR-NC22 }^{2} \\
\text { Transfection }\end{array}$ & - & - & + & - & - & + & - & - & + & - & - & + & - & - & + & - & - \\
\hline $\begin{array}{l}\text { miR-33b } \\
\text { Transfection }\end{array}$ & - & - & - & + & - & - & + & - & - & + & - & - & + & - & & + & - \\
\hline SREBF1 & 1- & & & & & & & & & & & & & & & -1 & 一 \\
\hline EBF1 & - & & - & & & & & & & & & & & & - & & - \\
\hline ACTB & - & & 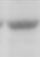 & & & - & & & & & & & & & & & ? \\
\hline
\end{tabular}
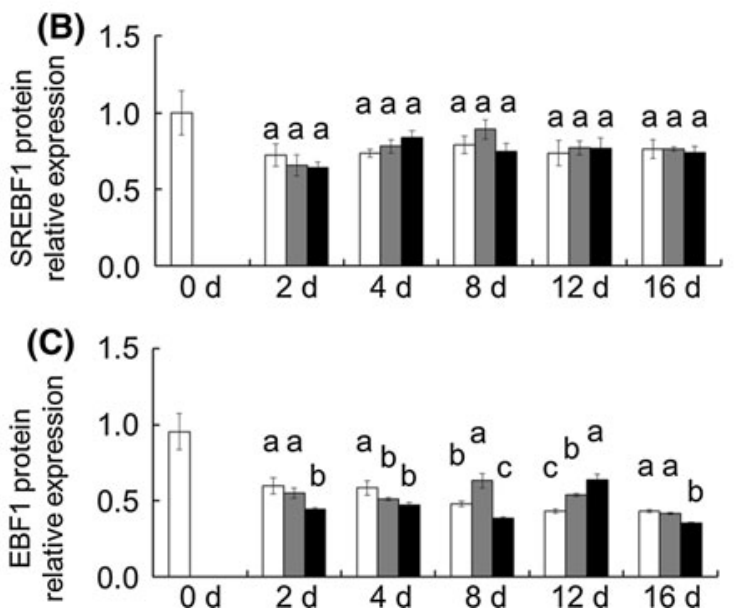

Fig. 4 Changes in SREBF1 and EBF1 protein expression levels in PSPA. PSPA were treated as described above. Relative gene expression was determined by Western blotting at least three times. The image shown is a representative result. Comparisons of relative protein expression were performed by using Tukey's multiple comparison tests. ${ }^{1} \mathrm{H}$ : Positive control (nucleoprotein extracted from HeLa cells). ${ }^{2}$ miR-NC: negative control miRNA. Different alphabets show a significant difference $(P<0.05)$

Therefore, we assumed that the observed decrease in lipogenesis in PSPA was caused by ssc-miR33b via the attenuation of adipogenic and lipogenic pathways that are regulated by $\mathrm{C} / \mathrm{EBP} \alpha$ and $\mathrm{PPAR} \gamma$, which are ordinarily activated by EBF1. We examined the promoter regions of the porcine PPAR $\gamma 1, \gamma 2$, and $C / E B P \alpha$ genes by using TFSERACH ver1.3 based on TRANSFAC [28] and found that the promoters of $C / E B P \alpha$ and $P P A R \gamma$ genes contained $\mathrm{EBF} 1$ and $\mathrm{C} / \mathrm{EBP} \alpha$ binding sites, respectively (Supplementary Figs. 3, 4, 5). These results suggest that porcine EBF1 may be associated with the transcriptional regulation of $\mathrm{C} / \mathrm{EBP} \alpha$ and, indirectly, of PPAR $\gamma$ genes.

\section{Effect of miR-33b on SREBF1 and EBF1 expression}

Transfection of precursor miRNAs into PSPA successfully induced the transitional expression of miR-33b. The expression level of miR-33b in miRNA precursor-transfected
PSPA was significantly higher $(P<0.05)$ than those in the control and in miR-NC-transfected PSPA, although the endogenous miR-33b level was relatively low and did not fluctuate over time (Fig. 3).

Because of the genomic localization of ssc-miR-33b, we examined whether miR-33b influences its host SREBF1 gene. SREBF1 gene expression did not appear to be elevated during the PSPA differentiation induction time course. The mRNA level of SREBFl upon miR-33b transfection tended to be lower throughout the time course, but no significant differences were detected (Fig. 3). In addition, the relative protein expression level of SREBF1 after miR-33b transfection was not different from that after transfection of miR-NC (Fig. 4). These results suggest miR-33b had relatively little effect on SREBF1 expression.

In contrast, the mRNA levels of $E B F 1$ in cells with miR-33b transfection were significantly $(P<0.05)$ decreased by $66.9 \%$ ( 4 days), $70.2 \%$ ( 8 days), and $80.8 \%$ (12 days) compared with those after miR-NC transfection (Fig. 3). In addition to this mRNA abundance, the protein expression level of EBF1 in miR-33b-transfected PSPA was significantly $(P<0.05)$ decreased by $80.8 \%$ (2 days), $61.2 \%$ (8 days), and $84.8 \%$ (16 days) compared with that after miR-NC transfection (Fig. 4). These results suggest that miR-33b affects EBF1 expression at both the transcriptional and post-transcriptional levels.

Effect of miR-33b on $C / E B P \alpha, P P A R \gamma$ and their downstream adipo/lipogenic genes

Given our finding of decreased EBF1 expression upon miR-33b transfection, we investigated the gene expression of the adipogenic and lipogenic master regulators $C / E B P \alpha$ and $P P A R \gamma$ and their downstream genes, including $a P 2$, CD36, ADIPOQ, SCD1, and FASN (Fig. 3).

The relative mRNA level of $P P A R \gamma 2$ gradually increased in the differentiation-induced PSPA. However, the PPAR 2 mRNA level after miR-33b transfection significantly decreased compared with that after miR-NC transfection from the 2-day time point onward $(P<0.05)$. In agreement with the PPAR 2 mRNA expression, the relative gene expression of $P P A R \gamma 1$, another isoform of $P P A R \gamma$ broadly expressed in various tissue types, was also decreased in miR-33b-transfected PSPA compared with that in miR-NC-transfected PSPA $(P<0.05)$. Similarly, the relative gene expression of $C / E B P \alpha$ in miR-33b-transfected PSPA was significantly decreased compared with that in miR-NC-transfected PSPA from the 2-day time point onward $(P<0.05)$. These results demonstrate that the changes in the expression patterns of $P P A R \gamma$ and $\mathrm{C} / E B P \alpha$ were similar, although the levels of gene expression were different. The results of the gene expression assays for $P P A R \gamma 2$ and $C / E B P \alpha$ suggest that the decrease 
Fig. 5 Comparison of miR$33 \mathrm{~b}$, lipogenic genes, and fat formation indices between Meishan- and Landrace-derived crossbred gilts. Expression of miR-33b and lipogenic genes in muscle (LD muscle), liver and $\mathrm{Sc}$ fat (subcutaneous fat) tissues. Open squares and shaded circles indicate individual crossbred gilts derived from Landrace (EL) and Meishan (EM), respectively. Mean values are denoted with horizontal bars
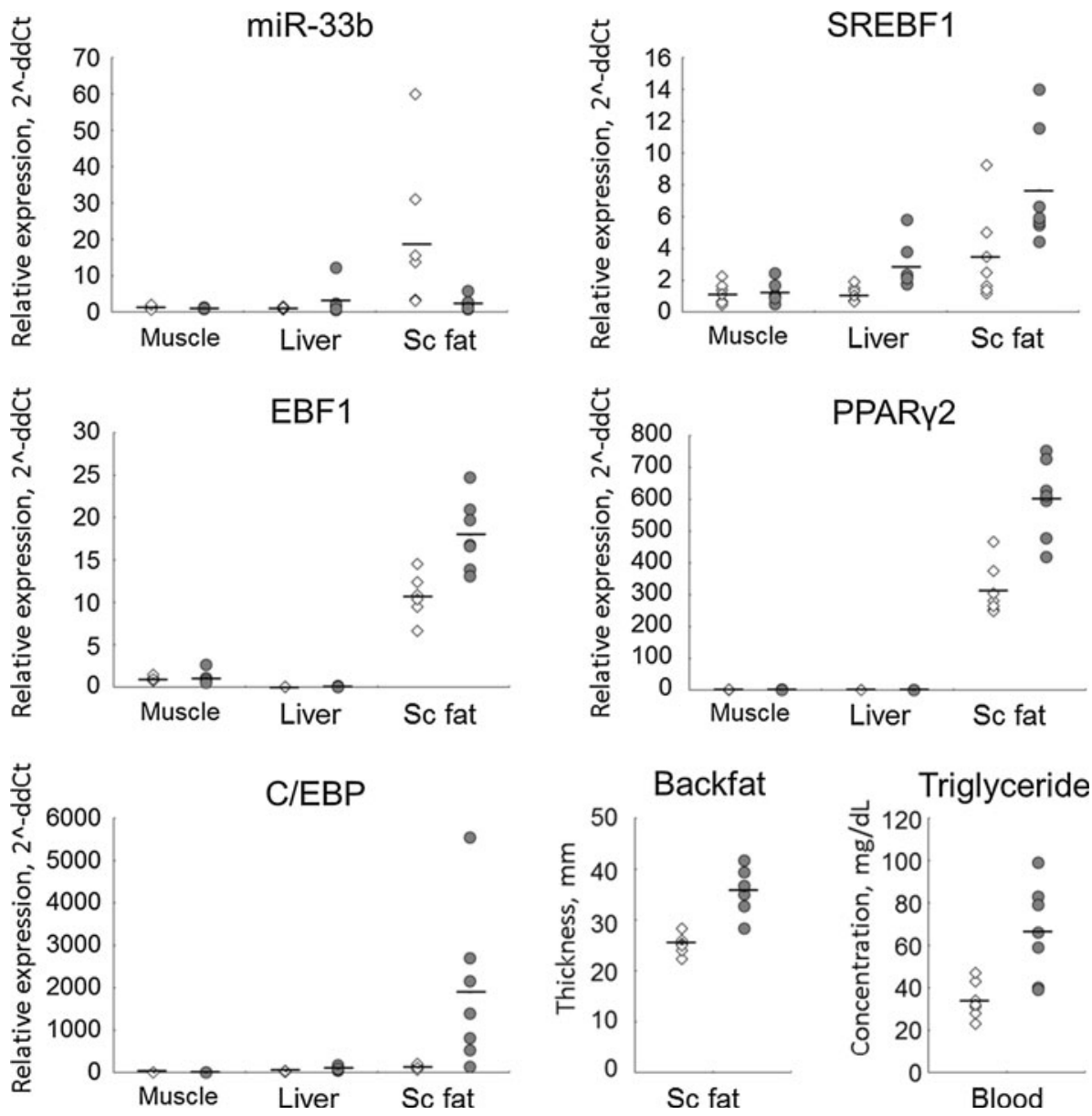

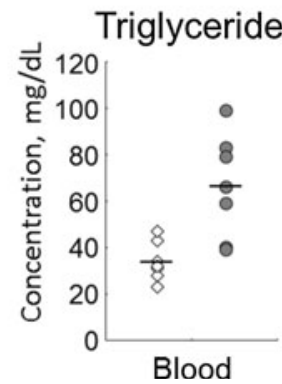

in expression of these genes was affected by EBF1 degradation, as indicated in the BioSystems Database of the NCBI and by Jimenez et al. [26].

Genes known to be regulated by PPAR $\gamma$, such as $a P 2$, $C D 36$, and $A D I P O Q$, were highly expressed in adipose differentiation-induced PSPA, whereas their expression was significantly decreased in miR-33b-transfected PSPA (Fig. 3). In addition, the same fluctuating expression pattern was observed for the PPAR 2 gene, suggesting that the decreased expression of the $a P 2, C D 36$, and $A D I P O Q$ genes was the result of decreased PPAR $\gamma 2$ in miR-33btransfected PSPA. In human adipocytes, transcription of $a P 2, C D 36$, and $A D I P O Q$ is reported to be regulated by $\operatorname{PPAR} \gamma 2$, and these genes are associated with fat formation and metabolism [31-33].

The mRNA level of $S C D l$ was generally increased in line with the degree of lipogenesis in adipose differentiation-induced PSPA, but the mRNA level after miR-33b transfection was at least $30 \%$ less than that after miR-NC transfection from the 4-day time point onward $(P<0.05)$ (Fig. 3). The observed decrease in SCD1 mRNA expression levels was similar to that of the PPAR 2 mRNA level, because $S C D 1$ gene transcription can be regulated by
SREBF1 or PPAR $\gamma 2[5,34]$. The mRNA level of FASN after miR-33b transfection was $41 \%$ less than that after miR-NC transfection at 4 days $(P<0.05)$. In contrast to the $S C D 1$ mRNA level, changes in the FASN mRNA level were small, suggesting that $F A S N$ transcription may be modestly regulated by PPAR $\gamma 2$, as demonstrated in previous studies $[35,36]$.

Taken together, these results suggest that expression of adipogenic and lipogenic genes is well regulated by the master regulators PPAR $\gamma 2$ and C/EBP $\alpha$ in PSPA and that the observed decrease of lipid accumulation in PSPA can be explained by the decrease in expression of these key genes. Furthermore, the delay in adipogenesis and decrease in lipogenesis observed in PSPA after miR-33b transfection was characterized by the degradation of PPAR $\gamma 2$ and $\mathrm{C} / \mathrm{EBP} \alpha$ but not that of SREBF1, possibly due to decreased EBF1 transcriptional regulation.

Possible effect of miR-33b on fat formation differences between pig breeds

To investigate the effect of miR-33b on fat formation in fattening pigs and on the generation of different fat traits in 
pigs that were crossbred with typical lean (Landrace)- and fatty (Meishan)-type breeds, we analyzed differences in BF thickness and blood TG levels and also in miR-33b, $S R E B F 1, E B F 1, P P A R \gamma$, and $C / E B P \alpha$ gene expression in the subcutaneous tissues of EL and EM crossbred gilts.

We found that EM gilts showed significantly higher blood TG levels $(P<0.01)$ and BF thickness $(P<0.001)$ than did EL gilts, in agreement with a previous finding [37]. In addition to this difference in physiological characteristics between these pig breeds, gene expression assays indicated that miR-33b expression in EL tended to be higher than that in EM $(P=0.08)$ (Fig. 5). In contrast, EL gilts showed significantly lower expression of lipogenic genes, including SREBF1 $(P<0.01), E B F 1(P<0.01)$, $P P A R \gamma 2(P<0.01)$, and $C / E B P \alpha(P<0.05)$ than did EM gilts. These results suggest that the difference in miR-33b expression between these pig breeds is associated with differences in the transcriptional regulation of lipogenic genes, leading to observed differences in fat-related traits such as BF thickness and blood TG levels. It is important to note that only seven gilts were used to obtain these data; therefore, given the small sample size, it is difficult to draw conclusions regarding correlations between miR-33b and lipogenic gene regulation and fat-related traits. In addition, genetic polymorphisms in SREBF1 and miR-33b may have certain effect on their expressions, although so far there is no evidence for a difference in the SREBF1 gene sequences between Landrace and Meishan pigs. As described in a review by Dodson et al. [2], meat animals have recently begun to be utilized as good experimental models for lipid metabolic research. In addition, the lack of miR-33b in Srebfl gene in traditional model animals such as mouse and rat (Supplementary Fig. 1) $[11,12]$ may mean that the pig is a better model for biomedical research on lipid metabolism.

Future studies of miR-33b will include evaluating correlations between miR-33b, lipogenic genes, and pork carcass characteristics related to fat tissues by using a larger number of pigs.

Acknowledgments This work was supported by KAKENHI (22780256).

Open Access This article is distributed under the terms of the Creative Commons Attribution License which permits any use, distribution, and reproduction in any medium, provided the original author(s) and the source are credited.

\section{References}

1. Hishikawa D, Hong YH, Roh SG, Miyahara H, Nishimura Y, Tomimatsu A, Tsuzuki H, Gotoh C, Kuno M, Choi KC, Lee HG, Cho KK, Hidari H, Sasaki S (2005) Identification of genes expressed differentially in subcutaneous and visceral fat of cattle, pig, and mouse. Physiol Genomics 21(3):343-350

2. Dodson MV, Hausman GJ, Guan L, Du M, Rasmussen TP, Poulos SP, Mir P, Bergen WG, Fernyhough ME, McFarland DC, Rhoads RP, Soret B, Reecy JM, Velleman SG, Jiang Z (2010) Lipid metabolism, adipocyte depot physiology and utilization of meat animals as experimental models for metabolic research. Int J Biol Sci 6(7):691-699

3. Tontonoz P, Hu E, Spiegelman BM (1994) Stimulation of adipogenesis in fibroblasts by PPAR $\gamma 2$, a lipid-activated transcription factor. Cell 79(7):1147-1156

4. Tong Q, Dalgin G, Xu H, Ting CN, Leiden JM, Hotamisligil GS (2000) Function of GATA transcription factors in preadipocyteadipocyte transition. Science 290(5489):134-138

5. Shimano H (2001) Sterol regulatory element-binding proteins (SREBPs): transcriptional regulators of lipid synthetic genes. Prog Lipid Res 40(6):439-452

6. Le Lay S, Lefrère I, Trautwein C, Dugail I, Krief S (2002) Insulin and sterol-regulatory element-binding protein-1c (SREBP-1C) regulation of gene expression in 3T3-L1 adipocytes. Identification of CCAAT/enhancer-binding protein beta as an SREBP-1C target. J Biol Chem 277(38):35625-35634

7. Horton JD, Shah NA, Warrington JA, Anderson NN, Park SW, Brown MS, Goldstein JL (2003) Combined analysis of oligonucleotide microarray data from transgenic and knockout mice identifies direct SREBP target genes. Proc Natl Acad Sci USA 100(21):12027-12032

8. Bartel DP (2004) MicroRNAs: genomics, biogenesis, mechanism, and function. Cell 116(2):281-297

9. Kozomara A, Griffiths-Jones S (2011) miRBase: integrating microRNA annotation and deep-sequencing data. Nucl Acid Res 39(Database issue):D152-D157

10. Najafi-Shoushtari SH, Kristo F, Li Y, Shioda T, Cohen DE, Gerszten RE, Näär AM (2010) MicroRNA-33 and the SREBP host genes cooperate to control cholesterol homeostasis. Science 328(5985):1566-1569

11. Rayner KJ, Suárez Y, Dávalos A, Parathath S, Fitzgerald ML, Tamehiro N, Fisher EA, Moore KJ, Fernández-Hernando C (2010) MiR-33 contributes to the regulation of cholesterol homeostasis. Science 328(5985):1570-1573

12. Dávalos A, Goedeke L, Smibert P, Ramírez CM, Warrier NP, Andreo U, Cirera-Salinas D, Rayner K, Suresh U, Pastor-Pareja JC, Esplugues E, Fisher EA, Penalva LO, Moore KJ, Suárez Y, Lai EC, Fernández-Hernando C (2011) miR-33a/b contribute to the regulation of fatty acid metabolism and insulin signaling. Proc Natl Acad Sci USA 108(22):9232-9237

13. Li G, Wu Z, Li X, Ning X, Li Y, Yang G (2011) Biological role of microRNA-103 based on expression profile and target genes analysis in pigs. Mol Biol Rep 38(7):4777-4786

14. Romao JM, Jin W, He M, McAllister T, Guan LL (2012) Altered microRNA expression in bovine subcutaneous and visceral adipose tissues from cattle under different diet. PLoS ONE 7(7): e40605

15. Legault C (1985) Selection of breeds, strains and individual pigs for prolificacy. J Reprod Fertil Suppl 33:151-166

16. White BR, Lan YH, McKeith FK, Novakofski J, Wheeler MB, McLaren DG (1995) Growth and body composition of Meishan and Yorkshire barrows and gilts. J Anim Sci 73(3): 738-749

17. Suzuki K, Asakawa S, Iida M, Shimanuki S, Fujishima N, Hiraiwa H, Murakami Y, Shimizu N, Yasue H (2000) Construction and evaluation of a porcine bacterial artificial chromosome library. Anim Genet 31(1):8-12

18. PE Applied Biosystems (1997) ABI prism 7700 sequence detection system: relative quantitation of gene expression. User Bull 2: 16-24 
19. Livak KJ, Schnittgen TD (2001) Analysis of relative gene expression data using realtime PCR and the 2[-Delta Delta $\mathrm{C}(\mathrm{t})$ ] method. Methods 25(4):402-408

20. Hoashi S, Ashida N, Ohsaki H, Utsugi T, Sasazaki S, Taniguchi M, Oyama K, Mukai F, Mannen H (2007) Genotype of bovine sterol regulatory element binding protein-1 (SREBP-1) is associated with fatty acid composition in Japanese Black cattle. Mamm Genome 18(12):880-886

21. Nakajima I, Muroya S, Chikuni K (2003) Growth arrest by octanoate is required for porcine preadipocytes differentiation. Biochem Biophys Res Commun 309(3):702-708

22. Kertesz M, Iovino N, Unnerstall U, Gaul U, Segal E (2007) The role of site accessibility in microRNA target recognition. Nat Genet 39(10):1278-1284

23. Lewis BP, Burge CB, Bartel DP (2005) Conserved seed pairing, often flanked by adenosines, indicates that thousands of human genes are microRNA targets. Cell 120(1):15-20

24. Betel D, Wilson M, Gabow A, Marks DS, Sander C (2007) The microRNA.org resource: targets and expression. Nucl Acids Res 36(Database issue):D149-D153

25. Geer LY, Marchler-Bauer A, Geer RC, Han L, He J, He S, Liu C, Shi W, Bryant SH (2004) The NCBI BioSystems database. Nucl Acids Res 38(Database issue):D492-D496

26. Jimenez MA, Åkerblad P, Sigvardsson M, Rosen ED (2007) Critical role for Ebf1 and Ebf2 in the adipogenic transcriptional cascade. Mol Cell Biol 27(2):743-757

27. Rosen ED, Spiegelman BM (2001) PPARgamma: a nuclear regulator of metabolism, differentiation, and cell growth. J Biol Chem 276(41):37731-37734

28. Hausman GJ (2000) The influence of dexamethasone and insulin on expression of CCAAT/enhancer binding protein isoforms during preadipocytes differentiation in porcine stromal-vascular cell cultures: evidence for very early expression of C/EBPalpha. J Anim Sci 78(5):1227-1235

29. Desvergne B, Michalik L, Wahli W (2006) Transcriptional regulation of metabolism. Physiol Rev 86(2):465-514
30. Heinemeyer T, Wingender E, Reuter I, Hermjakob H, Kel AE, Kel OV, Ignatieva EV, Ananko EA, Podkolodnaya OA, Kolpakov FA, Podkolodny NL, Kolchanov NA (1998) Databases on transcriptional regulation: TRANSFAC, TRRD, and COMPEL. Nucl Acids Res 26(1):362-367

31. Rival Y, Stennevin A, Puech L, Rouquette A, Cathala C, Lestienne F, Dupont-Passelaigue E, Patoiseau JF, Wurch T, Junquéro D (2004) Human adipocyte fatty acid-binding protein (aP2) gene promoter-driven reporter assay discriminates nonlipogenic peroxisome proliferator-activated receptor gamma ligands. J Pharmacol Exp Ther 311(2):467-475

32. Combs TP, Wagner JA, Berger J, Doebber T, Wang WJ, Zhang BB, Tanen M, Berg AH, O'Rahilly S, Savage DB, Chatterjee K, Weiss S, Larson PJ, Gottesdiener KM, Gertz BJ, Charron MJ, Scherer PE, Moller DE (2002) Induction of adipocyte complement-related protein of 30 kilodaltons by PPARgamma agonists: a potential mechanism of insulin sensitization. Endocrinology 143(3):998-1007

33. Rać ME, Safranow K, Poncyljusz W (2007) Molecular basis of human CD36 gene mutations. Mol Med 13(5-6):288-296

34. Tabor DE, Kim JB, Spiegelman BM, Edwards PA (1999) Identification of conserved cis-elements and transcription factors required for sterol-regulated transcription of stearoyl-CoA desaturase 1 and 2. J Biol Chem 274(29):20603-20610

35. Kim JB, Sarraf P, Wright M, Yao KM, Mueller E, Solanes G, Lowell BB, Spiegelman BM (1998) Nutritional and insulin regulation of fatty acid synthetase and leptin gene expression through ADD1/SREBP1. J Clin Invest 101(1):1-9

36. Hogan JC, Stephens JM (2005) The regulation of fatty acid synthase by STAT5A. Diabetes 54(7):1968-1975

37. Nakajima I, Oe M, Ojima K, Muroya S, Shibata M, Chikuni K (2011) Cellularity of developing subcutaneous adipose tissue in Landrace and Meishan pigs: adipocyte size differences between two breeds. Anim Sci J 82(1):144-149 